\section{Atypical Haemolytic Uraemic Syndrome Associated with Cabazitaxel in Advanced Prostate Cancer}

\section{Abstract}

Atypical haemolytic uraemic syndrome, (aHUS) is a rare but potentially lifethreatening condition with clinical manifestations of haemolytic anaemia, thrombocytopenia, and renal failure. We report the first case of aHUS secondary to cabazitaxel in the setting of metastatic castrate resistant prostate cancer. In July 2015, a 73 year old male presented with acute on chronic back pain, in the context of metastatic castrate-resistant prostate cancer with known pulmonary and skeletal metastases. He was day 3 into his first cycle of cabazitaxel. Within 24 hours of admission, he acutely deteriorated with progressive drowsiness and the development of an acute kidney injury. During this period, he developed acute thrombocytopenia, falling to $58 \times 10^{9} / \mathrm{L}$ and haemolytic anaemia. He had a coagulopathy, with an elevated INR 1.5, but with a normal APTT and fibrinogen levels. He was subsequently diagnosed with an atypical haemolytic uraemic syndrome. In light of his rapid decline and poor prognosis he was managed with conservative treatment. Over the next 24 hours, he developed progressive acute renal failure and ongoing haemolysis. There was further neurological deterioration which culminated in his passing away three days following his initial presentation. With the recent advent of various synthetic therapies, including cabazitaxel, it is more imperative for clinicians to be aware of potentially life threatening adverse reactions, especially if irreversible.

Keywords: Cabazitaxel; Atypical Haemolytic Uraemic Syndrome (aHUS); Prostate cancer; Renal failure

\author{
Abishek Tumma ${ }^{1}$ and \\ Shu Fen Lee ${ }^{2}$ \\ Department of Medicine, Princess
Alexandra Hospital, Australia
2 Department of Medical Oncology,
Princess Alexandra Hospital, Australia
}

Corresponding author: Abishek Tumma

abishek_tumma@hotmail.com

Department of Medicine, Princess

Alexandra Hospital, Australia

Tel: 064435158371

Citation: Tumma A, Lee SF. Atypical Haemolyltic Uraemic Syndrome Associated with Cabazitaxel in Advanced Prostate Cancer. Med Case Rep. 2016, 2:3.

\section{Introduction}

Atypical haemolytic uraemic syndrome (aHUS) is an uncommon, but potentially life threatening disease with a high mortality rate [1]. It is typically characterised by complement dysfunction leading to thrombocytopaenia, haemolytic anaemia and acute renal failure [2] However, almost $20 \%$ of cases are associated with extra-renal manifestations, including diabetes mellitus, cardiomyopathy, cerebrovascular events, hepatic failure and myopathy [3]. Although aHUS comprises of only $10 \%$ of haemolytic uraemic cases, these patients unfortunately have poor outcomes [4]. Previous estimates have suggested mortality rates ranging between 10 to $40 \%$, with up to $50 \%$ of patients progressing to end stage renal failure $[5,6]$. There are many triggers for developing aHUS, which also include connective tissue disease, medications, malignancy and chemotherapy. Chemotherapeutic agents previously associated with aHUS include bleomycin, mitomycin C, cisplatin, fluorouracil, fludarabine and gemcitabine. In particular, the mortality related to chemotherapy-induced aHUS is largely due to renal failure. We present the first case of cabazitaxel related aHUS with a review of the literature surrounding aHUS and its association with chemotherapy and prostate cancer.

\section{Case Report}

On the $16^{\text {th }}$ July 2015 , a 73 year old male presented with acute on chronic back pain, in the context of metastatic castrate-resistant prostate cancer with known pulmonary and skeletal metastases. His MRI whole spine, performed on the day of presentation revealed the source of pain to be secondary to progressive skeletal metastases at the level of L3 and was planned for palliative radiation. He was day 2 into his first cycle of cabazitaxel. His routine biochemistry and full blood count blood tests performed 2 days prior to the cabazitaxel dose were unremarkable, except for a chronic mild normocytic anaemia. 
The patient was initially diagnosed with locally advanced prostate cancer in 2011 based on an elevated PSA level, imaging results and digital rectal examination findings and was commenced on intermittent androgen deprivation therapy with goserelin. In 2014, he developed urinary obstruction and underwent a transurethral resection of the prostate. Biopsy at that time confirmed a Gleason $4+5=9$ prostate adenocarcinoma. Re-staging scans then revealed new metastatic disease to the bone and lung and he was started on maximal androgen blockade with the addition of bicalutamide. He unfortunately had disease progression in late 2014 and was commenced on docetaxel chemotherapy. He had poor tolerance of docetaxel and was switched to enzalutamide after two cycles. Regrettably, he did not respond to enzalutamide with further disease progression after three cycles and subsequently developed haemoptysis. He was given a single fraction of radiation to his right pulmonary hilar region to control his haemoptysis and then started on his third line of systemic therapy with cabazitaxel at $25 \mathrm{mg} / \mathrm{m}^{2}$. His PSA at the time was $250 \mu \mathrm{g} / \mathrm{L}$.

On the $18^{\text {th }}$ July, day 4 following his first dose of cabazitaxel, he acutely deteriorated with progressive drowsiness and the development of an acute kidney injury with a creatinine of $375 \mu \mathrm{mol} / \mathrm{L}$ and urea of $30.8 \mathrm{mmol} / \mathrm{L}$ (Table 1). There was also an acute decrement in his platelet count, falling to $58 \times 10^{9} / \mathrm{L}$ with associated normocytic anaemia with fragmented red cells seen on peripheral blood film. His haemolytic screen confirmed a low haptoglobin at $0.05 \mathrm{~g} / \mathrm{L}$, however his Direct Coombs test was negative. Coagulation studies revealed an elevated INR of 1.5 whilst both his APTT and fibrinogen levels were normal. He was reviewed by both haematology and nephrology and was diagnosed with aHUS. In light of his rapid decline and poor prognosis with limited treatment options available for his underlying metastatic prostate cancer, he was deemed inappropriate for plasmapharesis, dialysis or eculizumab.

Over the $19^{\text {th }}$ July, his creatinine climbed to $519 \mu \mathrm{mol} / \mathrm{L}$ with a urea of $44.8 \mathrm{mmol} / \mathrm{L}$ and a further drop in his haemoglobin and platelet count to $82 \mathrm{~g} / \mathrm{L}$ and $59 \times 10^{9} / \mathrm{L}$, respectively. There was further neurological deterioration on the same day which culminated in his passing away.

\section{Discussion}

HUS is a disease entity on the spectrum of microangiopathic haemolytic anaemia. It is characterised by anaemia, thrombocytopaenia and acute renal failure. The diagnosis of HUS requires the exclusion of a severely low ADAMTS13

Table 1: Development of acute kidney injury with creatinine.

\begin{tabular}{|c|c|c|c|c|c|}
\hline & \multicolumn{3}{|c}{ Pre Cabazitaxel } & \multicolumn{4}{c|}{ Post Cabazitaxel } \\
\hline & $3^{\text {rd }}$ July & $13^{\text {th }}$ July & $16^{\text {th }}$ July & $18^{\text {th }}$ July & $19^{\text {th }}$ July \\
\hline Creatinine $(\mu \mathrm{mol} / \mathrm{L})$ & 67 & 68 & 79 & 375 & 519 \\
\hline $\begin{array}{c}\text { Urea }(\mathrm{mmol} / \mathrm{L}) \\
\text { eGFR }\end{array}$ & 7.8 & 10 & 10.7 & 30.8 & 44.8 \\
\hline $\begin{array}{c}\text { (CKD-EPI) } \\
\text { Haemoglobin }(\mathrm{g} / \mathrm{L})\end{array}$ & 114 & 105 & 102 & 105 & 82 \\
\hline \begin{tabular}{c} 
Platelets $\left(\times 10^{9} / \mathrm{L}\right)$ \\
\hline LDH $(\mathrm{U} / \mathrm{L})$
\end{tabular} & 204 & 219 & 182 & 58 & 59 \\
\hline $\begin{array}{c}\text { ePSA }(\mu \mathrm{g} / \mathrm{L}) \\
\text { (ref }<6.5)\end{array}$ & 612 & 104 & - & 2730 & 3090 \\
\hline & 108 & 180 & 250 & - & - \\
\hline
\end{tabular}

level (activity <5\%) which is more characteristic of thrombotic thrombocytopaenic purpure [7] Although associated with thrombocytopaenia, only $50 \%$ of patients with HUS have an initial platelet count of below $100 \times 10^{9} / \mathrm{L}$. The median haemoglobin level in these patients was found to be approximately $80 \mathrm{~g} / \mathrm{L}$ [8].

The pathophysiology behind HUS has been thought to be secondary to complement mediated destruction of vascular endothelium of arterioles or capillaries. This effect results in platelet aggregation and activation of the coagulation cascade leading to microangiopathic thrombi consisting of platelet and fibrin. Indeed, histological sections of the kidneys, which are primarily affected, commonly reveal micro-thrombi occluding the glomerular capillaries and afferent arterioles, resulting in marked glomerular sclerosis, hypocellularity, necrosis and interstitial fibrosis. Biochemically, it is associated with haemolytic anaemia which is characterised by a low haptoglobin level, elevated bilirubin and lactate dehydrogenase as well as erythrocyte fragmentation on a peripheral blood smear.

HUS is divided into either typical or atypical forms. The typical form is often related to an infective diarrheal illness usually associated with the Shiga-like toxin secreted by Escherichia coli. However, other organisms such as shigella and campylobacter have also been implicated. These toxins directly disrupt normal complement activity leading to microangiopathic haemolytic anaemia. The atypical form (aHUS) is related to underlying defects in the complement system and is triggered by causes such as collagen vascular disease, transplantation, pregnancy, malignancy and medications including chemotherapy. In support of this theory, $50 \%$ of aHUS patients are found to have an underlying genetic complement defect.

It can be a challenge to discriminate the underlying cause of aHUS between underlying malignancy and chemotherapy [9]. Literature suggests malignancy related HUS occurs in widespread metastatic disease or poorly controlled carcinomas. Whereas chemotherapy associated HUS is more common in situations of disease remission or in the presence of minimal tumour burden. The prevalence of microangiopathic haemolytic anaemia in malignancy is between $3.5-7.8 \%[10,11]$ There have been over 150 reported cases in the literature of HUS associated with solid malignancy, especially in gastric, breast, lung, prostate and cancer of unknown primary. Of these cases, little more than 20 reports have been associated with prostate cancer [12] Furthermore, it is interesting to note that over $91 \%$ of cancers were metastatic on diagnosis of aHUS. The most common site of metastases was bone. In the cases of prostate cancer as described, PSA levels have generally ranged between 59 to $>1000 \mathrm{ng} / \mathrm{ml}[13,14]$ Once treated, the recurrence rate of the syndrome in prostate cancer is low. However, despite chemotherapy and surgery, median survival for prostate cancer patients with aHUS is only 10.5 months.

To date, chemotherapeutic agents previously associated with aHUS include bleomycin, mitomycin C, cisplatin, fluorouracil, fludarabine and gemcitabine. The most frequently reported agent is still mitomycin- $C$, with the incidence ranging between $2-10 \%$ [15]. Although the onset of haemolytic anaemia can be immediately after therapy, it more commonly occurs 4-8 weeks after exposure to the causative agent. Proposed mechanisms for chemotherapy induced aHUS have been thought to be secondary 
to direct endothelial damage, leading to platelet aggregation and intravascular haemolysis [16].

In this reported case, although the patient had progressive metastatic disease, the development of symptoms within several days of commencement of cabazitaxel suggests a potential chemotherapy-induced cause. However, as demonstrated in our patient, this discrimination is not always clear. There has been some suggestion that the level of serum factors such as tumour necrosis factor-alpha, interleukin-1, interleukin-6, von Willebrand factor (VWF) antigen and low molecular weight vWF multimer may be able to distinguish between these two causes [17-19]. These are however still in the experimental phase, and efforts are limited by the paucity of cases.

The management of HUS is controversial. Regardless of cause, the overall treatment is largely supportive with the management of renal failure with dialysis if required. There may also be a potential role for eculizumab which will be discussed later. In general, although plasmapharesis reduces rates of haemolysis it does not significantly reduce renal failure, and is therefore not recommended. Upwards of $80 \%$ of patients with aHUS have partial responses to short-term plasmapharesis limited to improvement in platelet count and haemoglobin levels. Over $50 \%$ of cases continue to progress to end stage renal failure despite plasmapharesis [20].

The specific management for aHUS differs between whether the underlying cause is either chemotherapy or malignancy-related. In chemotherapy-induced cases, treatment involves withdrawal of the causative drug. For malignancy-induced cases, management primarily relies on early initiation of therapy targeting the underlying disease, which would be chemotherapy in most cases. The haematologic response of aHUS to chemotherapy may be quick, with the potential for normalisation of blood counts after only one cycle. Treatment with plasmapharesis is rarely effective in malignancy, except in cases of prostate cancer. The good response rate of plasmapharesis in prostate cancer may suggest a possible underlying antibody-mediated mechanism. However, no such antibodies have been found as yet [21]. Eculizumab, a humanised monoclonal $c 5$ inhibitor has been studied in several open label phase II studies and has been associated with a significant reduction in the progression of renal disease and requirement for plasmapharesis [22]. Eculizumab has been used as rescue therapy in many forms of secondary hemolytic uraemic syndrome including post-bone marrow transplantation [23]. However, its role in malignancy or chemotherapy induced aHUS has not been defined [24,25].

Despite the availability of treatments, the outcome with aHUS is poor with an associated high mortality, ranging from 10 to $40 \%$, and up to $50 \%$ of patients progressing to end stage renal failure. Most patients with malignancy associated HUS died with or without treatment within one month. ${ }^{12}$ In comparison, mortality related to cases of chemotherapy induced HUS has been largely attributed to renal failure, rather than progression of underlying malignancy [26].

\section{Conclusion}

HUS is a potentially life threatening complication that has been previously well described as secondary to multiple chemotherapeutic agents. We report the case of a patient who developed aHUS following his first cycle of cabazitaxel. This is a rare but serious adverse effect that would require vigilance.

\section{Conflict of Interest}

The authors note there is no potential conflict of interest relevant to this article. 


\section{References}

1 Noris M (2010) Relative role of genetic complement abnormalities in sporadic and familial aHUS and their impact on clinical phenotype. Clin J Am Soc Nephrol 5: 1844-1859.

2 Norris M, Remuzzi G (2015) Hemolytic uremic syndrome. Journal of American Society of Nephrology 16: 1035.

3 Hofer J, Rosales A (2014) Extra-renal manifestations of complementmediated thrombotic microangiopathies. Frontiers in Pediatrics 2: 97.

4 Westra D, Wetzels J (2012) A new era in the diagnosis and treatment of atypical haemolytic uraemic syndrome. The Netherlands Journal of Medicine 70.

5 Kavanagh D, Goodship T, Richards A (2006) Atypical haemolytic uraemic syndrome. Br Med Bull 77: 5 -22.

6 Saif W, McGee J (2005) Hemolyltic-uremic syndrome associated with gemcitabine: A case report and review of literature. J Pancreas 6: 369-374.

7 Bianchi V, Robles R (2002) Von Willebrand factor-cleaving protease (ADAMTS13) in thrombocytopenic disorders: a severely deficient activity is specific for thrombotic thrombocytopenic purpura. Blood 100.

8 Corrigan J, Boineau F (2001) Hemolytic-uremic syndrome. Pediatrics in Review 22.

9 Gordon I, Kwaan C (1997) Cancer and drug- associated thrombotic thrombocytopenic purpura and hemolytic uremic syndrome. Semin Hematol 34: 140-147.

10 Vesely K, George N, Lammle B, Studt D, Alberio L, et al. (2003) ADAMTS13 activity in thrombotic thrombocytopenic purpurahemolytic uremic syndrome: relation to presenting features and clinical outcomes in a prospective cohort of 142 patients. Blood 102: 60-68.

11 Levandovsky M, Harvey D, Lara P, Wun T (2008) Thrombotic thrombocytopenic purpura-hemolytic uremic syndrome (TTP-HUS): A 24-year clinical experience with 178 patients. J Hematol Oncol 1: 23.

12 Lechner K, Obermeier L (2012) Cancer-related microangiopathic hemolytic anaemia. Medicine 91.

13 Basic-Jukic N, Kes P (2007) Treatment of thrombotic microangiopathies with plasma exchange. Hematology 12: 63-67.

14 Biers S, Sullivan M (2004) Thrombotic microangiopathy in advanced prostatic carcinoma. Urology 63.
15 Gradishar J, Vokes E, Ni K, Panje R (1990) Chemotherapy-related hemolytic uremic syndrome after the treatment of head and neck cancer: a case report. Cancer 66: 1914-1918.

16 Ustwani O, Lohr J, Dy G (2014) Eculizumab therapy for gemcitabine induced hemolytic uremic syndrome: case series and concise review. Journal of Gastrointestinal Oncology 5.

17 Melnyk A, Solez K, Kjellstrand C (1995) Adult hemolytic-uremic syndrome. A review of 37 cases. Arch Intern Med 155: 2077-2084.

18 Hillyer D, Duncan A, Ledford M, Barrett J, Klumpp A, et al. (1995) Chemotherapy- induced hemolytic uremic syndrome: description of a potential animal model. J Med Primatol 24: 68-73.

19 Monteagudo J, Pereira A, Roig S, Reverter JC, Ordinas A, et al. (1990) Investigation of plasma von Willebrand factor and circulating platelet aggregating activity in mitomycin C-related hemolytic-uremic syndrome. Am J Hematol 33: 46-49.

20 Verhave J, Wetzels J, Van De Kar N (2014) Novel aspects of atypical haemolytic uraemic syndrome and the role of eculizumab. Ephrol Dial Transplant 29.

21 Dragon-Durey M, Blanc C, Garnier A (2010) Anti-factor H autoantibody-associated hemolytic uremic syndrome: review of literature of the autoimmune form of HUS. Semin Thromb Hemost 36: 633 .

22 Legendre C, Licht C (2013) Terminal complement inhibitor eculizumab in atypical hemolytic-uremic syndrome. The New England Journal of Medicine 368: 2169-2181.

23 Peffault De Latour R, Xhaard A (2013) Successful use of eculizumab in a patient with post-transplant thrombotic microangiopathy. British Journal of Haematology 161: 279-280.

24 Schrezenmeier H, Hochsmann B (2012) Drugs that inhibit complement. Transfus Apher Sci 46: 87-92.

25 Weitz M, Amon O, Brassler D, Koenigsrainer A, Nadalin S (2011) Prophylactic eculizumab prior to kidney transplantation for atypical hemolytic uremic syndrome. Pediatr Nephrol 26: 1325-1329.

26 Wu Dou, Ming Liu (1997) Mitomycin-C Induced hemolytic uremic syndrome: A case report and literature review. Jpn J Clin Oncol 27: 115-118. 